\title{
Clinical Practice Guidelines
}

International Myeloma Working Group (IMWG) recommendations for the treatment of relapsed and refractory multiple myeloma

\section{Tables: 3}

References: 62

Words : 5888

\section{Introduction}

The treatment of multiple myeloma (MM) has changed dramatically in the past decade with the incorporation of new drugs into therapeutic strategies. These drugs, in various combinations, have been added to national and international clinical guidelines, and have transformed our approach to the treatment of patients with MM, resulting in a significant improvement in overall survival (OS). ${ }^{1,2}$

With the availability of at least seven different classes of approved agents, i.e., alkylators, steroids, proteasome inhibitors (PIs), immunomodulatory agents (IMiDs), histone deacetylase inhibitors (HDACi), monoclonal antibodies (MoAbs) and selective inhibitors of nuclear export (SINEs) that can be combined in doublet, triplet or even quadruplet regimens, and used together with or without high-dose therapy and autologous stem cell transplantation (ASCT), or in some cases as continuous treatment, the choice of the optimal strategy at diagnosis and at relapse represents a challenge for physicians. Moreover, soon, next generation immunotherapies or targeted agents, such as venetoclax, will improve the therapeutic armamentarium. Also problematic is the lack of trials addressing important questions, such as the integration of the first salvage regimen into the assessment of front-line therapies in order to define optimal sequencing strategies in homogeneous patient populations. Furthermore, there is a considerable lack of information on the efficacy of the different approved regimens on specific patient populations such as refractory 
disease versus treatment of relapse occurring after a treatment-free interval, biochemical versus symptomatic relapse, relapse after oneprior line versus more advanced disease, high-risk versus standard risk cytogenetics, etc. ${ }^{3}$

Multiple phase 3 trials have demonstrated improved survival outcomes, both progression free survival (PFS) and OS with the use of triplet combinations suggesting that at least two active drugs should be combined with steroids if patients can tolerate this safely. However, combinations of the new agents mentioned above are regrettably associated with a high cost, raising de facto two important issues: drug access in both emerging and developed countries, and the definition of value vs patient benefit.

At the time of relapse, the treatment choice is influenced by many patient- and disease-related factors, such as age, cytogenetics, pre-existing toxicities, comorbidities, aggressiveness of the relapse, patient choice, but mostly by the type and the response to the previous therapies. ${ }^{4}$ The aim of this manuscript is to review currently available data for the treatment of relapsed and refractory MM (RRMM), and propose clear recommendations for routine practice.

\section{Treatment of patients with relapsed/refractory disease who have received one prior line of therapy}

Overall, the most important question in the majority of the cases is whether a patient is refractory to lenalidomide or not. A second scenario, that will be increasingly important is whether the patient is progressing on frontline therapies that include daratumumab. Both scenarios will be discussed. Finally, the role of salvage ASCT will also be discussed.

Lenalidomide is part of frontline therapy in newly-diagnosed MM (NDMM), based on OS benefits seen in randomized trials and meta-analyses. In patients treated with upfront ASCT, lenalidomide single agent at low dose is approved as maintenance therapy until progression. ${ }^{5-6}$ In patients with previously untreated NDMM, who are not eligible for ASCT, lenalidomide is also approved in combination with low-dose dexamethasone $(\mathrm{Rd})$ until disease progression, based on the results of the FIRST study. ${ }^{7}$ In addition, in the prospective SWOG0777 trial, which enrolled patients with NDMM who were not intended to undergo immediate ASCT, the regimen of Rd plus 
bortezomib (VRd) followed by Rd until progression resulted in significantly improved PFS and OS.8,9 Therefore, there is a high number of patients with disease progressing on continuous lenalidomide.

\section{First relapse in patients with lenalidomide-refractory disease (Table 1)}

Patients with lenalidomide-refractory disease were rightly excluded from recent randomized phase 3 trials testing $R d$ vs $R d$ plus a third agent (either a PI, [carfilzomib, ${ }^{10} \mathrm{KRd}$, or ixazomib, ${ }^{11} \mathrm{IRd}$ ] or a MoAb, [elotuzumab, ${ }^{12}$ Elo-Rd, or daratumumab, ${ }^{13} \mathrm{DRd}$ ). The exact role of lenalidomide-based triplet combinations in patients refractory to lenalidomide is unknown, however, most likely they would lead to suboptimal results, and these regimens are therefore rarely used in this setting. The only study available showing that the addition of a third agent to lenalidomide and steroids may rescue lenalidomide-refractory disease is a phase 1/2 trial reported by the HOVON group. ${ }^{14}$ In 67 patients, Nijhof et al. showed that the addition of continuous low-dose oral cyclophosphamide $(50 \mathrm{mg} / \mathrm{d})$ to $25 \mathrm{mg}$ lenalidomide and prednisone (REP regimen) induced a $67 \%$ response rate, with a median PFS and OS of 12.1 and 29 months, respectively, in lenalidomide-refractory patients.

For a patient progressing on lenalidomide as part of frontline therapy, the logical approach is a switch in the class of agent, from an IMiD to a PI.

Bortezomib-dexamethasone ( $\mathrm{Vd}$ ) was the first combination used in this setting, resulting in a PFS ranging from 8 to 10 months. ${ }^{15}$ Cyclophosphamide may also be added to $\mathrm{Vd}(\mathrm{VCd})$ to increase the response rate, but no prospective comparison of $\mathrm{Vd}$ versus $\mathrm{VCd}$ in relapsed myeloma is available.

Several phase 3 trials have evaluated PI-based combinations using $\mathrm{Vd}$ as control arm in RRMM, but few truly lenalidomide-refractory patients were included. Four trials can be discussed in this setting.

First, in the phase 3 randomized ENDEAVOR study Vd was prospectively compared to carfilzomib-dexamethasone $(\mathrm{Kd})$ until progression in the relapse setting in patients with one to three prior lines of therapy. ${ }^{16,17}$ This study, a head-to-head comparison of two PIs, demonstrated that both PFS (median 18.7 versus 9.4 months) ${ }^{16}$ and OS (median 47.6 versus 40 months) ${ }^{17}$ were superior with $\mathrm{Kd}$ across the whole group of patients. In this trial, the number of patients refractory to lenalidomide regardless of 
the number of prior lines of therapy was 113 for the $\mathrm{Kd}$ arm and 122 for $\mathrm{Vd}$ arm, while the exact number of patients progressing on frontline lenalidomide is unknown. ${ }^{18}$ The median PFS for the group of lenalidomide-refractory patients was rather short, 8.6 and 6.6 months with $\mathrm{Kd}$ and $\mathrm{Vd}$, respectively, ${ }^{18}$ and there was a not significant improvement of 7.8 month with $\mathrm{Kd}$ vs $\mathrm{Vd}$ regarding survival (median OS, 29.2 versus 21.4 months; hazard ratio $[\mathrm{HR}]=0.857,95 \%$ confidence interval $[\mathrm{Cl}] 0.623-1.178) .{ }^{19}$ These findings suggest that patients with lenalidomide-refractory disease may not benefit as much from $\mathrm{Kd}$ as patients whose disease responds well to prior lenalidomide.

In another study, Vd was compared to Vd plus daratumumab (DVd) in patients with relapsed MM who had received at least one prior line of therapy (CASTOR trial). ${ }^{20}$ The triplet combination was associated with a significant PFS improvement (median not reached versus 7.2 months; HR 0.39$)^{20}$, which was confirmed in an updated analysis, in which, after a median follow-up of 19.4 months, the median PFS for DVd was 16.7 versus 7.1 months for Vd alone (HR 0.31). ${ }^{21}$ As in the ENDEAVOR study, the number of patients progressing on frontline lenalidomide is unknown. The only information available is based on a sub-analysis, showing that patients refractory to lenalidomide, regardless of the number of prior lines of therapy, (DVd, $n=60 ; \mathrm{Vd}, \mathrm{n}=$ 81) also achieved a significant PFS benefit with DVd versus Vd, with medians of 7.8 versus 4.9 months, respectively, not very different from the data reported in the ENDEAVOR study for a similar subgroup of patients which suggest that DVd is also suboptimal for this population. ${ }^{22}$ Overall survival data for this subgroup of patients are not available to date. Importantly, the safety profile of the triplet combination is acceptable, and daratumumab was not found to add any significant toxicity to the $\mathrm{Vd}$ combination.

The phase 3 PANORAMA 1 study, comparing $V d$ vs $V d+$ panobinostat, enrolled a subgroup of patients progressing on lenalidomide as frontline therapy, but the number of patients in this setting was very small and prior treatment with lenalidomide was not a stratification factor. ${ }^{23}$ Overall, the study showed that the combination of $\mathrm{Vd}+$ panobinostat improved PFS by four months, but did not result in an OS benefit. ${ }^{24}$ The toxicity observed in the panobinostat arm of the trial, especially the high frequency of grade 3-4 gastro-intestinal adverse events, fatigue and 
thrombocytopenia, does not argue in favor of the use of this triplet combination in lenalidomide-refractory patients.

Recently, in the phase 3 OPTIMISMM trial, which was reported in 2019, the combination of pomalidomide plus $\mathrm{Vd}$ (PomVd, $\mathrm{n}=278$ ) was prospectively compared to $\mathrm{Vd}(\mathrm{n}=270)$ in patients with RRMM who had received one to three prior lines of therapy that included lenalidomide. ${ }^{25}$ More than $70 \%$ of the patients were refractory to lenalidomide. After a median follow-up of 16 months, PomVd demonstrated an improved median PFS (11.2 versus 7.1 months; HR 0.61; $\mathrm{P}<0.0001$ ). The median PFS was also prolonged with PomVd in patients refractory to lenalidomide (9.5 versus 5.6 months, $P=0.0008)$, in patients with one previous line of treatment (20.7 versus 11.6 months, $\mathrm{P}=0.0027$ ) and particularly interesting were the results in patients who had received one previous line of treatment and were refractory to lenalidomide (17.8 versus 9.5 months, $P=0.03$ ). Overall survival data are lacking due to the relatively short follow-up (16.4 months).

The combinations of Kd plus CD38 antibodies have recently been evaluated in phase 3 studies. In the CANDOR trial, $\mathrm{Kd}$ was prospectively compared with $\mathrm{Kd}+$ daratumumab (Dara-Kd) in RRMM patients who had received one to three prior lines of therapy (446 patients, $33 \%$ lenalidomide refractory). ${ }^{26}$ This study showed that the median PFS was not reached for the Dara-Kd group and it was 15.8 months for the $K d$ group (HR 0.63, $P=0.0027$ ). Dara-Kd was superior to $K d$ in terms of PFS both among lenalidomide-exposed (HR 0.53) and lenalidomide-refractory patients (HR 0.47). Furthermore, both the ORR ( $84 \%$ versus $75 \%, P=0.008$ ) and the minimal residual disease $(M R D)$ negative rate at 12 months $(13 \%$ versus $1 \%, P<0.0001)$ were in favor of Dara-Kd. In the phase 3 IKEMA trial, which was reported at EHA 2020 meeting, 302 patients with RRMM and 1-3 prior lines of therapy were randomized to receive either Isatuximab plus $K d$ (Isa-Kd, $n=179)$ or $K d(n=123) .{ }^{27}$ At a median follow-up of 20.7 months, median PFS was not reached for Isa-Kd vs 19.1 months for $\mathrm{Kd}$ (HR 0.53; $\mathrm{P}=0.0007$ ). Isa-Kd was superior to $\mathrm{Kd}$ in terms of PFS both among lenalidomide-exposed (HR 0.50) and lenalidomide-refractory patients (HR 0.60). Dara-Kd and Isa-Kd are not yet approved by the regulatory authorities, but, based on PFS data and HRs, may be considered as important options for first relapse in patients with lenalidomide-refractory disease in the near future. 
Lenalidomide-exposed patients have been studied in few phase $1 \mathrm{~b} / 2$ trials, which evaluated new combinations based on Pls and/or pomalidomide +/- MoAbs. Major limitations are the small number of patients, as well as the short follow-up, and lack of OS data. Jakubowiak et al. reported data of a phase 2 randomized trial comparing $\mathrm{Vd}$ versus Vd + elotuzumab in 152 patients with RRMM, showing a PFS benefit of the triplet combination in the intent-to-treat population (9.7 versus 6.9 months). ${ }^{28}$ Sixty-six percent of the patients were treated at the time of the first relapse, but the number of cases progressing on lenalidomide is not reported, and a subgroup analysis of patients previously treated with IMiDs showed no PFS benefit of the addition of elotuzumab to Vd (HR 0.87, 95\% Cl 0.56-1.34).

In the phase 1b MMY1001 trial, EQUULEUS, one arm tested the combination of pomalidomide-dexamethasone plus daratumumab (D-Pd). ${ }^{29}$ Ninety-two patients out of 102 enrolled into this arm had lenalidomide-refractory disease. The ORR for the whole group of patients was $66 \%$, and the median PFS was 10.1 months after a median follow-up of 28.1 months. However, the number of patients progressing on frontline lenalidomide therapy included in this arm was very small. The same combination, D-Pd, was investigated in a phase 2 trial (MM-014) conducted in North America and involved 112 patients who had progressed after lenalidomide-based therapy (median two prior lines), 84 ( $75 \%$ ) of whom were refractory to lenalidomide. ${ }^{30}$ With a median follow-up of 8.2 months, the ORR (primary end-point) was $75 \%$ in lenalidomide-refractory patients, and the 9-month PFS rate was $86.3 \%$ (range 76.5 92.2\%), while the median PFS was not estimable. ${ }^{30}$ Pomalidomide was also combined with twice-weekly $56 \mathrm{mg} / \mathrm{m} 2$ carfilzomib and dexamethasone (KPd) in the prospective EMN011/HO114 trial conducted by the European Myeloma Network. ${ }^{31}$ This phase 2 trial was designed for patients with refractory disease or first progression after having received therapy as part of the EMN02 trial, in which patients were randomized to frontline ASCT versus no frontline ASCT followed by consolidation and lenalidomide maintenance until progression. After four 28-day cycles of re-induction with KPd, patients were offered either salvage ASCT, if they had not received frontline intensive therapy, or four additional cycles of $\mathrm{KPd}(8 \mathrm{KPd}$ cycles overall). Subsequently, patients with stable disease or better received pomalidomide $4 \mathrm{mg}$ with or without dexamethasone in 28 days cycles until progression. ${ }^{31}$ The analysis of the first 60 patients, 57 (95\%) of whom had 
progressed on lenalidomide maintenance, showed that responses to $\mathrm{KPd}$ were quick, with a median time to best response of two months. The toxicity of KPd was manageable, and at a median follow-up of 16.3 months, the median PFS was 18 months, with a better outcome in patients with standard-risk cytogenetics (HR 0.27) and in patients, who had not received frontline ASCT ( $n=25$, HR 0.49). Pom-dex has also been combined with oral weekly ixazomib (IPD) and tested in a phase I/II trial in a group of 32 lenalidomide-refractory patients with a median of two prior lines of therapy (range 1-5). ${ }^{3}$ The exact number of patients progressing on frontline lenalidomide is unknown. The ORR was $48 \%$, and the median PFS was 8.6 months. This triplet all oral combination was well-tolerated, and some patients above the age of 80 years were enrolled (up to 84 years).

Other important phase 3 studies in RRMM are ongoing, Pomalidomidedexamethasone +/- daratumumab (APPOLO/EMN14, NCT03180736), Vd +/venetoclax (BELLINI, NCT02755597) or Vd +/- selinexor (BOSTON, NCT03110562), and the first results are expected in 2020. Nevertheless, a common limitation of these studies is that they include patients with varying degree of refractoriness to frontline lenalidomide are included and will therefore provide only subgroup analyses.

\section{Recommendations for first relapse in patients with lenalidomide-refractory disease}

Preferred options (based on phase 3 trials): Pom-Vd or Dara-Kd or Isa-Kd (grade of recommendation $[1 \mathrm{~A}])$

Second options (based on phase 3 trials): Dara-Vd, Kd; [1B]

Other options (based on phase 2 trials): KPd, Pd-dara; IPd in frail; [1C]When daratumumab-isatuximab / carfilzomib / pomalidomide not available: VCd, Vd, VMP

\section{First relapse in patients with disease not refractory to lenalidomide (Table 2)}

In patients who have received a bortezomib-based therapy upfront (i.e., VCD, VTD, VMP) without lenalidomide maintenance, or patients treated with a fixed duration of lenalidomide with a progression occurring more than 6 months after stopping therapy, second line therapy should be based on lenalidomide- and dexamethasone-based regimens, i.e. $\mathrm{Rd}$ in combination with carfilzomib (KRd), ${ }^{10}$ daratumumab (DaraRd), ${ }^{13}$ 
ixazomib (IRd) ${ }^{11}$ or elotuzumab (EloRd) ${ }^{12}$. In pivotal phase 3 studies, all of these combinations were found to be superior to Rd regarding PFS, the primary end-point for these trials. $\mathrm{KRd}^{33}$ and Elo-Rd ${ }^{34}$, investigated in the two trials with the longest follow-up, also showed an OS benefit compared to Rd for the intent-to treat patient population.

The most effective combination available to date in the setting of first relapse not refractory to lenalidomide is $\mathrm{DRd}^{12}$. In the POLLUX trial, DRd was shown to significantly prolong PFS in the intent-to-treat population compared to Rd (median 44.5 vs 17.5 months; HR $0.44,95 \% \mathrm{Cl} 0.35-0.55, \mathrm{P}<0.0001$ ) after a median of 44.3 months follow-up, and in the subgroup of patients who had received one prior line of therapy, DRd $(n=149)$ also significantly prolonged PFS versus $R d(n=146$; median 53.3 vs 19.6 months; HR $0.42,95 \% \mathrm{Cl} 0.31-0.58, \mathrm{P}<0.0001) .{ }^{35}$ Median PFS2 was not reached with D-Rd versus 31.7 months with $\mathrm{Rd}(\mathrm{HR} 0.53,95 \% \mathrm{Cl} 0.42-$ $0.68, \mathrm{P}<0.0001)$ in the intent-to-treat population. ${ }^{35}$ These results are expected to translate into an OS benefit with longer follow-up. The DRd triplet combination is well tolerated, and the forthcoming availability of a subcutaneous mode of administration of daratumumab will increase convenience. ${ }^{36}$ In the ASPIRE trial, the median OS was 11.4 months longer for $K R d(n=184)$ versus $R d(n=157)$ in patients who had received one prior line of therapy (47.3 versus 35.9 months; $\mathrm{HR} 0.81,95 \% \mathrm{Cl} 0.62$ to 1.06). ${ }^{33}$ Elo-Rd and IRd are well tolerated, but less effective. The OS benefit observed with Elo-Rd vs Rd is restricted to patients with 2-3 prior lines of therapy, and OS is similar in patients with 1 prior line of therapy (median 43.7 versus 44.1 months, HR 1, 95\% Cl 0.77-1.32). ${ }^{34}$

After frontline therapy based on combinations including a $\mathrm{PI}$, a retreatment including a PI may also be considered. Two trials, ENDEAVOR ${ }^{16}$, evaluating carfilzomib 56 $\mathrm{mg} / \mathrm{m} 2$ twice weekly, and $\mathrm{CASTOR}^{20}$, evaluating DVD, have shown superiority vs VD. In ENDEAVOR, patients previously exposed to frontline bortezomib were enrolled if they were not refractory to bortezomib. The median PFS for patients who had received one prior line of therapy ( $K d, n=231 ; V d, n=229)$ was 22.2 months for $\mathrm{Kd}$ versus 10.1 months for $\mathrm{Vd}^{18}$ and when patients had previously received bortezomib, the median PFS for Kd was 15.6 versus 8.1 months for Vd (HR: 0.56, 95\% Cl: 0.44-0.73). The median OS in patients treated after one prior line was 51.3 versus 43.7 months, respectively (HR $0.77,95 \% \mathrm{Cl} 0.58-1.02) .{ }^{19}$ In CASTOR, after 
19.4 months of median follow up, DVD was found to prolong PFS compared to VD alone (median 16.7 versus 7.1 months; HR $0.31,95 \% \mathrm{Cl} 0.24-0.39, \mathrm{P}<0.0001$ ). The PFS benefit of DVD was most apparent in patients with one prior line of therapy (median 27 versus 7.9 months; HR $0.22,95 \% \mathrm{Cl} 0.13-0.33$; $\mathrm{P}<0.0001$ ). ${ }^{21}$ The phase 3 BOSTON trial reported at ASCO 2020 has compared VD vs VD plus selinexor (SVD) weekly, in 402 patients who had received 1-3 prior therapies. ${ }^{37}$ SVD significantly prolonged median PFS vs VD (13.9 vs 9.4 months, $H R=0.70, P=0.0066$ ), but this benefit was less apparent in patients previously exposed to a PI (HR 0.78, $95 \% \mathrm{Cl}$ 0.58-1.06). SVd has not been licensed yet. Finally, the results of the CANDOR trial, in which KD was compared to Dara-KD, showed a trend for a longer PFS for patients with one prior line of therapy (HR $0.68,95 \% \mathrm{Cl} 0.40-1.14$ ), those not refractory to lenalidomide ( $\mathrm{HR} 0.74,95 \% \mathrm{Cl} 0.49-1.11)$, and those with prior $\mathrm{PI}$ exposure (HR $0.61,95 \% \mathrm{Cl} 0.45-0.84) .{ }^{25}$ Similarly, the preliminary results of the IKEMA trial, in which KD was compared to Isa-KD, also showed a trend for a longer PFS for patients with one prior line of therapy (HR 0.59, 95\% Cl 0.31-1.12), those not refractory to lenalidomide ( $\mathrm{HR} 0.45,95 \% \mathrm{Cl} 0.15-1.35)$, and those with prior $\mathrm{PI}$ exposure (HR 0.56, 95\% $\mathrm{Cl} 0.31-1.04) .{ }^{27}$

Recommendations for first relapse in patients with disease not refractory to lenalidomide

Preferred options (based on phase 3 trials): DRD or KRD when DRD not available; [1A]Second options (based on phase 3 trials): DVD, KD, Dara-KD, Isa-KD, IRd, Elo$\mathrm{Rd}$; [1B]When daratumumab-isatuximab / carfilzomib not available: Rd, Vd, VCD, VTD, VMP

First relapse in patients progressing on daratumumab-based combinations frontline

The approval of daratumumab-based regimens as first line therapy in myeloma (DaraVMP,D-VMP [ALCYONE trial] ${ }^{38-39}$ and DaraRd [MAIA trial] ${ }^{40}$ ), is making decisions regarding treatment lines challenging. To date, there is no data for daratumumab retreatment at second line, and salvage therapy using isatuximab in patients progressing on daratumumab is an unlikely option. 
In the ALCYONE trial, patients in the D-VMP group received nine 6-week cycles of subcutaneous bortezomib, oral melphalan and oral prednisone, plus intravenous daratumumab until disease progression or unacceptable toxicity. ${ }^{38}$ At a median follow-up of 40.1 months, a significant benefit in OS was observed for the D-VMP group, compared with the VMP group (HR 0.60, 95\% Cl 0.46-0.80, $\mathrm{P}=0.0003$ ). ${ }^{39}$ The Kaplan-Meier estimate of the 36-month rate of OS was $78.0 \%(95 \% \mathrm{Cl} 73.2-82.0)$ in the D-VMP group and $67.9 \%(95 \% \mathrm{Cl} 62.6-72.6)$ in the VMP group. No data are available yet regarding subsequent therapies after D-VMP failure. Nevertheless, at the time of relapse, the logical approach is to use a lenalidomide-based combination, without daratumumab. ALCYONE enrolled elderly patients not eligible for ASCT. An attractive option would be KRD for fit elderly patients in this setting, but for frail or over the age of 75 years, $\mathrm{Rd}$ alone or in combination with ixazomib or elotuzumab might be the best approaches following progression on D-VMP.

In the MAIA trial, patients received RD-daratumumab frontline until progression. ${ }^{40}$ This combination is now approved, and the impressive PFS results will lead to a widespread use of this triplet combination, even in patients older than 75 years. No data on salvage regimens at the time of progression in the MAIA trial are available. A PI-based combination without daratumumab is the logical approach. In this setting, KD, VCD, PVD, VMP or KPD are reasonable options; alternatively, VD-Elo, SVD, or IPD can be considered.

\section{Salvage ASCT}

Frontline ASCT is the standard of care for fit patients less than 71 years of age in many countries. ${ }^{1-2}$ Nevertheless, given the lack of OS benefit of front-line ASCT in patients with standard risk disease compared to VRD followed by lenalidomide maintenance for example, ${ }^{41}$ some investigators and patients prefer to delay ASCT to the time of the first relapse, after harvesting and storing stem cells during induction. In this setting, salvage ASCT should be systematically considered in patients who never received a transplant before. ${ }^{2}$ One issue is the selection of the optimal reinduction regimen prior to salvage ASCT, especially for patients progressing on lenalidomide that was given upfront as long-term therapy. Only few data are available regarding re-induction regimens. KPD was found to be active in this setting in a phase 2 study conducted by the HOVON group. ${ }^{31}$ 
Salvage ASCT may also be considered in patients progressing after frontline ASCT. The only randomized controlled study to demonstrate the role of salvage ASCT in myeloma patients at first relapse/progression at least 12 months after prior ASCT was the UK Myeloma X study. ${ }^{42-43}$ In this study, relapsing MM patients who achieved at least stable disease after PAD re-induction (bortezomib/ doxorubicin/dexamethasone) had an improved time to disease progression (19 months versus 11 months, HR $0.45, P<0.0001)^{42}$ and OS (67 months versus 52 months, HR 0.56, $P=0.022)^{43}$ with salvage ASCT $(n=89)$ versus weekly oral cyclophosphamide $(n=85)$ as consolidation (probably a suboptimal scheme). Another prospective phase 3 study recently compared continuous $\mathrm{Rd}$ versus $\mathrm{Rd}$ re-induction followed by ASCT and maintenance with lenalidomide in patients with $1^{\text {st }}$ to $3^{\text {rd }}$ relapse in 277 patients, of which 260 had 1 line of prior therapy at the time of study entry, and $93 \%$ did receive frontline ASCT. ${ }^{44}$ Median PFS was 20.7 months in the transplant arm and 18.8 in the continuous Rd arm (HR 0.87, $p=0.34$ ), and median OS was not reached in the transplant arm and 62.7 months in the control arm (HR $0.81, p=0.37$ ), showing a marginal benefit for salvage ASCT.

The most important prognostic factor for PFS after salvage ASCT is the duration of remission following the first ASCT procedure. Since frontline ASCT followed by lenalidomide maintenance is associated with a median duration of response of 50 months ${ }^{41}$, one should not recommend salvage ASCT in patients with a response duration of less than three years after the first ASCT, but this cut-off is arbitrary and could be reduced to 2 years if the patient has not received maintenance therapy. [2A]

\section{Treatment of patients with relapsed/refractory disease who have received two or more prior lines of therapy (Table 3)}

The treatment of patients who have received two or more prior lines of therapy is becoming particularly challenging. Lenalidomide and bortezomib are commonly used as part of frontline therapy or at first relapse. Monoclonal antibodies and carfilzomib are also increasingly used during the first two lines of treatment. Therefore, at the time of the second relapse, all of the agents listed for use at first relapse can be used 
that have not been tried so far. A clinical trial, when available, should always be considered.

Few phase 3 trials have focused on patients who have received two or more prior lines of therapy. In patients whose disease has progressed following treatment with bortezomib and lenalidomide, pomalidomide plus dexamethasone (Pom-dex) has been considered as standard of care, following the results of the MM-003 randomized study. ${ }^{45}$ This combination has recently been compared to Pom-dex plus isatuximab (IsaPd) in the ICARIA trial in patients previously treated with two or more lines of therapy including lenalidomide and a PI. ${ }^{46}$ Of note, $92 \%$ of patients were refractory to lenalidomide, and $98 \%$ were refractory to the last line of therapy. At a median followup of 11.6 months, the median PFS (primary end-point) was 11.5 months in the IsaPd group $(n=154)$ versus 6.5 months in the Pd group $(n=153)$ (HR 0.596, $\mathrm{P}=0.001) .{ }^{46}$ IsaPd was recently approved by the Food and Drug Administration (FDA) and EMA in this setting. The CANDOR study (discussed above), in which Kd was compared to Dara-Kd, also included a pre-specified analysis of the outcome of 266 patients who had received two or more prior lines of therapy; the evaluation showed that HR for PFS was $0.61(95 \% \mathrm{Cl} 0.45-0.84)$, in favor of the triplet combination. ${ }^{26}$ Similarly, the IKEMA trial study, in which Kd was compared to Isa-Kd looked at the outcome of 167 patients who had received two or more prior lines of therapy; the analysis showed that HR for PFS was 0.48 (95\% Cl 0.29-0.78), in favor of Isa-KD. ${ }^{27}$

Two other antibody-based combinations, which can be considered for patients with advanced disease, have been approved based on phase 2 trials. In the randomized phase 2 study ELOQUENT-3, patients who had received at least two prior therapies were randomly assigned to receive either EloPd ( $n=60)$ or $\mathrm{Pd}(n=57) .47$ After a followup period of 9 months, the median PFS was 10.3 months in the EloPd group versus 4.7 in the $\mathrm{Pd}$ group ( $\mathrm{HR}$ 0.54, $\mathrm{P}=0.008) .{ }^{47}$ The combination of daratumumab, pomalidomide and dexamethasone (DaraPd) has also been licensed by the FDA for patients whose disease has failed at least two lines of previous therapies, including lenalidomide and a PI. This was based on a phase 2, non-randomized study, the EQUULEUS trial, in which DaraPd was given to 103 patients with RRMM. ${ }^{29}$ At a median follow-up of 13 months, the median PFS was 8.8 months and the median OS was 17.5 months. ${ }^{29}$ The ongoing phase 3 APOLLO study, designed to compare PD 
versus PD-Dara, is enrolling patients refractory to lenalidomide and PIs and will add important information for the treatment of patients beyond two lines of therapy.

A simple and cheap option to improve on the results of PD when other agents are not available is the addition of cyclophosphamide. Although no direct phase 3 comparisons are available, several phase 2 trials have shown that the median PFS of PCD is approximately 7-9 months, as compared with 4-6 months for the same subgroup of patients treated with PD alone. ${ }^{48}$

Additional options

For patients whose disease has become refractory to PIs, IMiDs, and CD38 antibodies, the outcome is very poor. A recent study revealed that these patients have a median OS of 5.6 months only. ${ }^{49}$

In this setting, intensive "old" combinations, such as VDT-PACE can be used, ${ }^{50}$ but no data are available in penta-refractory patients.

Selinexor, a SINE compound that blocks exportin 1 and forces nuclear accumulation and activation of tumor suppressor proteins, has been evaluated in combination with dexamethasone in patients who had previous exposure to bortezomib, carfilzomib, lenalidomide, pomalidomide, daratumumab, and an alkylating agent and had disease refractory to at least one $\mathrm{PI}$, one IMiD, and daratumumab (triple-class refractory) in the phase 2 STORM study. ${ }^{51} \mathrm{~A}$ total of 122 patients were included (53\% had highrisk cytogenetic abnormalities). A partial response or better was observed in $26 \%$, the median PFS was 3.7 months, and the median OS was 8.6 months. ${ }^{46} \mathrm{~A}$ pre-specified subgroup analysis of 83 patients whose disease was refractory to bortezomib, carfilzomib, lenalidomide, pomalidomide and daratumumab showed an ORR of $25.3 \%$, and the median response duration was 3.8 months. Based on these results, the FDA granted accelerated approval to selinexor for the treatment of this subgroup of patients in July 2019. One issue with this oral agent is the safety profile since one fourth of the patients experienced grade 3 fatigue, gastro-intestinal toxicity and thrombocytopenia.

As discussed previously, panobinostat, an oral pan-deacetylase inhibitor, was approved in combination with VD based on the results of PANORAMA 1 trial, ${ }^{23-24}$ but is scarcely used, due to an unfavorable safety profile, and lack of OSbenefit. Nevertheless, the phase 2 PANORAMA 2 trial showed that panobinostat was able to 
revert bortezomib resistance in one fourth of the cases progressing on bortezomibdexamethasone. ${ }^{52}$ Therefore, when patients are progressing on Pls, with few therapeutic options available, one may test the addition of panobinostat, with careful dose adaptation.

Promising investigational options

Melflufen is a first-in-class anti-cancer peptide-drug conjugate that rapidly delivers an alkylating payload into tumor cells. This agent has been tested in combination with dexamethasone in the phase1/2 O12-M1 trial in RRMM patients who had received two or more previous lines of therapy (including lenalidomide and bortezomib) and were refractory to their last line of therapy. In the phase 2 portion of the study, patients treated with melflufen plus dexamethasone achieved an overall response rate of $31 \% .^{53}$ The most common grade 3-4 adverse events were thrombocytopenia (62\% patients) and neutropenia (58\%), and non-hematological toxicity was infrequent. This promising agent is not approved yet, and the ongoing HORIZON trial is testing this combination in a large number of patients refractory to either pomalidomide and/or daratumumab.

BCMA-targeted therapies

B-cell maturation antigen (BCMA) promotes $\mathrm{MM}$ pathogenesis in the bone marrow microenvironment and is a very specific MM target antigen. Immunologically-based therapies targeting BCMA demonstrate promise independent of the genetic heterogeneity and genetic risk even in MM patients with no other treatment options. ${ }^{54}$ These agents include antibody-drug conjugates (ADCs), autologous chimeric antigen receptor engineered T cells (CAR-T), and bispecific $T$ cell engager. Very few data are yet available with bispecific agents, and early clinical trials are ongoing. ${ }^{54}$

Belantamab mafodotin is anti-BCMA ADC auristatin immunotoxin. In the DREAMM-2 phase 2 study, 196 patients with triple-class refractory MM received two different doses of belantamab mafodotin $(2.5 \mathrm{mg} / \mathrm{kg}[\mathrm{n}=97]$ or $3.4 \mathrm{mg} / \mathrm{kg}[\mathrm{n}=99]) .{ }^{55}$ Overall response was $31 \%$ and $34 \%$ for the two doses, respectively. The median PFS was 2.9 months in the $2.5 \mathrm{mg} / \mathrm{kg}$ cohort, and 4.9 months in the $3.4 \mathrm{mg} / \mathrm{kg}$ cohort, while OS data were not mature at the time of publication in December 2019. ${ }^{55}$ The most common grade $3-4$ adverse events included keratopathy $(27 \%$ and $21 \%$ of patients for the two doses, respectively), thrombocytopenia and anemia. ${ }^{55}$ Of note, in the 
phase 1 study, at the dose of $3.4 \mathrm{mg} / \mathrm{kg}$, the median PFS seemed longer (12 months), and the ORR was $60 \%$, but less patients had disease refractory to CD38 antibodies compared with the phase 2 study. ${ }^{56}$ Belantamab mafodotin is not yet approved by the authorities, but several countries may have access to this ADC in compassionate use programs.

Early clinical trials of CAR-T cell therapy have shown encouraging results in MM. In a phase 1 study of bb2121, a BCMA-targeting CAR T-cell construct, 33 of the 36 enrolled patients received CAR-T cells after lymphodepleting chemotherapy. ${ }^{57}$ Three patients progressed during manufacturing, which was successful in all patients. A total of 26 patients (79\%) were refractory to both a PI and an IMiD; 6 patients (18\%) were refractory to bortezomib, carfilzomib, lenalidomide, pomalidomide, and daratumumab. The ORR was $85 \%$, including a complete response rate of $45 \%$. Of the 16 patients with a hematologic response, who were evaluated for MRD, 15 were MRD-negative. For patients who received $\geq 150 \times 10^{6} \mathrm{CAR}-\mathrm{T}$ cells, the median PFS was 11.8 months. ${ }^{57}$ Cytokine release syndrome (CRS) occurred in $76 \%$ of the patients, and grade 3 or 4 neurotoxicity in $3 \%$ of the patients. The initial results of the phase 2 KarMMa study of idecabtagene vicleucel (ide-cel, bb2121) were reported at ASCO2020. ${ }^{58}$ One hundred and fourty patients were enrolled, of whom 128 were treated with ide-cel across the target dose levels of 150-450 x 106 CAR-T cells. All treated patients had been exposed to at least three prior therapies, including an IMiD, a PI and a CD38 antibody, and all were refractory to their last regimen. Eighty-four percent of patients were triple-refractory (refractory to an IMiD, PI and a CD38 antibody). With a median follow-up of 13.3 months, ORR and median PFS across the target dose levels $\left(150,300 \& 450 \times 10^{6}\right.$ CAR-T cells) were $73.4 \%$ (including $33 . \%$ complete response) and 8.8 months, respectively. ${ }^{58}$ These promising results have not yet been fully published, and ide-cel is not yet approved by the authorities. Results from a phase 1 study of LCAR-B38M CAR-T cells (LEGEND-2) conducted in China on 57 patients, less heavily pretreated (median of 3 prior lines of therapy), showed an ORR of $88 \%$, and a median PFS of 15 months. ${ }^{59}$ This second CAR T-cell construct targets two distinct BCMA epitopes derived from llama heavy chain antibodies, postulated to confer higher affinity toward BCMA. ${ }^{59}$ Ongoing trials in Europe and the US are using this construct. The phase 1b/2 CARTITUDE1 trial, using this agent, was reported at ASCO2020 in 29 patients, of which 25 were triple- 
class refractory. ${ }^{60}$ The overall response rate was $100 \%$, including $86 \%$ SCR, with a 9month PFS rate of $86 \%$. Cytokine release syndrome occurred in $93 \%$ of the patients (7\% grade $\geq 3$ ), and grade 3 or 4 neurotoxicity in $3 \%$ of the patients. Such promising results require confirmation on a larger number of patients. LCAR-B38M/JNJ-4528 is not approved by the authorities. Many other CAR-Ts are under evaluation targeting BCMA or other molecules, such as SLAMF7, CD38, NKG2D ligands, or CD138. ${ }^{61}$ The use of CAR-T cells raises several issues, especially in patients with very advanced disease: progression of the disease during product manufacturing, mechanisms of resistance (no plateau of PFS curves) related to antigen escape or lack of long-term persistence of CAR-T cells, safety (CRS and neurotoxicity). ${ }^{61}$

As mentioned previously, venetoclax, a selective BCL-2 inhibitor, was investigated in combination with $\mathrm{Vd}$ in the BELLINI phase 3 study. The study was stopped prematurely because of a reduced $O S$ in the Vd-venetoclax arm at the first interim analysis. Interestingly, a significant PFS benefit was reported with $\mathrm{Vd}$-venetoclax among patients with $\mathrm{t}(11 ; 14)(\mathrm{HR}=0.10,95 \% \mathrm{Cl}: 0.02-0.46, \mathrm{P}=0.003)$ and those with high bcl-2 expression ( $\mathrm{HR}=0.26,95 \% \mathrm{Cl}: 0.13-0.51, \mathrm{P}<0.001) .{ }^{62} \mathrm{Vd}$-venetoclax was also superior to $\mathrm{Vd}$ in terms of PFS (HR=0.26, 95\%Cl: $0.14-0.48, \mathrm{P}<0.001)$ and MRD negativity rate (19\% versus 0$)$ for the combined group of patients with $t(11 ; 14)$ or high bcl-2 expression. For this subgroup of patients, with a longer follow-up, venetoclax did not adversely impact OS. ${ }^{62}$ In the ongoing CANOVA phase 3 trial (NCT03539744), venetoclax plus dexamethasone is being compared to pomalidomide-dexamethasone in patients with RRMM with $t(11 ; 14)$ refractory to lenalidomide. This agent, not yet approved, may become the first targeted therapy for RRMM with either $\mathrm{t}(11 ; 14)$ and/or high bcl-2 expression.

Recommendations for patients with relapsed/refractory disease who have received two or more prior lines of therapy

\section{Preferred options:}

- Clinical trial

- Any first relapse options listed in tables $1 \& 2$ that have not been tried

- Isa-PD or Dara-KD (based on phase 3); [1 A]

Second options (based on phase 2): 
PD-Dara, Elo-Pd, KPd; [1B]When daratumumab / carfilzomib / elotuzumab not available: $P C D, P D$

\section{Other options:}

Approved: selinexor, addition of panobinostat to PIs, VTD-PACE

Investigational agents: melflufen, BCMA-targeting agents including CAR-Ts, ADC or bispecific antibodies, venetoclax in $\mathrm{t}(11 ; 14)$ or bcl-2 high expression 


\section{References}

1 - Moreau P, San Miguel J, Sonneveld P, Mateos MV, Zamagni E, Avet-Loiseau H et al. Multiple myeloma: ESMO Clinical Practice Guidelines for diagnosis, treatment and follow-up. Ann Oncol Off J Eur Soc Med Oncol 2017; 28: iv52-iv61.

2 - Kumar SK, Callander NS, Alsina M, Atanackovic D, Biermann JS, Castillo J et al. NCCN Guidelines Insights: Multiple Myeloma, Version 3.2018. J Natl Compr Cancer Netw JNCCN 2018; 16: 11-20.

3 - Moreau P, Rajkumar SV. Multiple myeloma--translation of trial results into reality. Lancet Lond Engl 2016; 388: 111-113.

4 - Moreau P. How I treat myeloma with new agents. Blood 2017; 130: 1507-1513.

5 - McCarthy PL, Holstein SA, Petrucci MT, Richardson PG, Hulin C, Tosi P et al. Lenalidomide Maintenance After Autologous Stem-Cell Transplantation in Newly Diagnosed Multiple Myeloma: A Meta-Analysis. J Clin Oncol Off J Am Soc Clin Oncol 2017; 35: 3279-3289.

6 - Jackson GH, Davies FE, Pawlyn C, et al. Lenalidomide maintenance versus observation for patients with newly diagnosed multiple myeloma (Myeloma XI): a multicentre, open-label, randomised, phase 3 trial. Lancet Oncol. 2019;20(1):57-73.

7 - Facon T, Dimopoulos MA, Dispenzieri A, Catalano JV, Belch A, Cavo M et al. Final analysis of survival outcomes in the phase 3 FIRST trial of up-front treatment for multiple myeloma. Blood 2018; 131: 301-310.

8 - Durie BGM, Hoering A, Abidi MH, Rajkumar SV, Epstein J, Kahanic SP et al. Bortezomib with lenalidomide and dexamethasone versus lenalidomide and dexamethasone alone in patients with newly diagnosed myeloma without intent for immediate autologous stem-cell transplant (SWOG S0777): a randomised, openlabel, phase 3 trial. Lancet Lond Engl 2017; 389: 519-527.

9 - Durie BGM, Hoering A, Sexton R, et al. Long-term follow-up of the randomized phase 3 trial SWOG S0777: bortezomib, lenalidomide and dexamethasone vs lenalidomide and dexamethasone in patients with previously untreated multiple myeloma without an intent for immediate autologous stem cell transplant. Blood Cancer J. 2020 May 11;10(5):53. doi: 10.1038/s41408-020-0311-8.

10 - Stewart AK, Rajkumar SV, Dimopoulos MA, Masszi T, Špička I, Oriol A et al. Carfilzomib, lenalidomide, and dexamethasone for relapsed multiple myeloma. N Engl J Med 2015; 372: 142-152.

11 - Moreau P, Masszi T, Grzasko N, Bahlis NJ, Hansson M, Pour L et al. Oral Ixazomib, Lenalidomide, and Dexamethasone for Multiple Myeloma. N Engl J Med 2016; 374: 1621-1634. 
12 - Lonial S, Dimopoulos M, Palumbo A, White D, Grosicki S, Spicka I et al. Elotuzumab Therapy for Relapsed or Refractory Multiple Myeloma. N Engl J Med 2015; 373: 621-631.

13 - Dimopoulos MA, Oriol A, Nahi H, San-Miguel J, Bahlis NJ, Usmani SZ et al. Daratumumab, Lenalidomide, and Dexamethasone for Multiple Myeloma. N Engl J Med 2016; 375: 1319-1331.

14 - Nijhof IS, Franssen LE, Levin M-D, Bos GMJ, Broijl A, Klein SK et al. Phase 1/2 study of lenalidomide combined with low-dose cyclophosphamide and prednisone in lenalidomide-refractory multiple myeloma. Blood 2016; 128: 2297-2306.

15 - Laubach J, Garderet L, Mahindra A, Gahrton G, Caers J, Sezer O et al. Management of relapsed multiple myeloma: recommendations of the International Myeloma Working Group. Leukemia 2016; 30: 1005-1017.

16 - Dimopoulos MA, Moreau P, Palumbo A, Joshua D, Pour L, Hájek R et al. Carfilzomib and dexamethasone versus bortezomib and dexamethasone for patients with relapsed or refractory multiple myeloma (ENDEAVOR): a randomised, phase 3 , open-label, multicentre study. Lancet Oncol 2016; 17: 27-38.

17 - Dimopoulos MA, Goldschmidt H, Niesvizky R, Joshua D, Chng W-J, Oriol A et al. Carfilzomib or bortezomib in relapsed or refractory multiple myeloma (ENDEAVOR): an interim overall survival analysis of an open-label, randomised, phase 3 trial. Lancet Oncol 2017; 18: 1327-1337.

18 - Moreau P, Joshua D, Chng W-J, Palumbo A, Goldschmidt H, Hájek R et al. Impact of prior treatment on patients with relapsed multiple myeloma treated with carfilzomib and dexamethasone vs bortezomib and dexamethasone in the phase 3 ENDEAVOR study. Leukemia 2017; 31: 115-122.

19 - Orlowski RZ, Moreau P, Niesvizky R, et al. Carfilzomib-dexamethsone versus bortezomib-dexamethasone in relapsed or refractory multiple myeloma: updated overall survival, safety, and subgroups. Clin. Lymphoma Myeloma Leuk. 2019;19:522-530.

20 - Palumbo A, Chanan-Khan A, Weisel K, Nooka AK, Masszi T, Beksac M et al. Daratumumab, Bortezomib, and Dexamethasone for Multiple Myeloma. N Engl J Med 2016; 375: 754-766.

21 - Spencer A, Lentzsch S, Weisel K, Avet-Loiseau H, Mark TM, Spicka I et al. Daratumumab plus bortezomib and dexamethasone versus bortezomib and dexamethasone in relapsed or refractory multiple myeloma: updated analysis of CASTOR. Haematologica 2018. doi:10.3324/haematol.2018.194118.

22 - Usmani SZ, Mateos M-V, Lentzsch S, Quach H, Capra M, Ovilla R et al. Efficacy of Daratumumab in Combination with Standard of Care Regimens in Lenalidomide- 
Exposed or -Refractory Patients with Relapsed/Refractory Multiple Myeloma (RRMM): Analysis of the Castor, Pollux, and MMY1001 Studies. In: Blood. 2018, pp 3288-3288.

23 - San-Miguel JF, Hungria VTM, Yoon S-S, Beksac M, Dimopoulos MA, Elghandour $A$ et al. Panobinostat plus bortezomib and dexamethasone versus placebo plus bortezomib and dexamethasone in patients with relapsed or relapsed and refractory multiple myeloma: a multicentre, randomised, double-blind phase 3 trial. Lancet Oncol 2014; 15: 1195-1206.

24 - San-Miguel JF, Hungria VTM, Yoon S-S, Beksac M, Dimopoulos MA, Elghandour $A$ et al. Overall survival of patients with relapsed multiple myeloma treated with panobinostat or placebo plus bortezomib and dexamethasone (the PANORAMA 1 trial): a randomised, placebo-controlled, phase 3 trial. Lancet Haematol 2016; 3: e506-e515.

25 - Richardson PG, Oriol A, Beksac $\mathrm{M}$ et al. Pomalidomide, bortezomib, and dexamethasone for patients with relapsed or refractory multiple myeloma previously treated with lenalidomide (OPTIMISMM): a randomised, open-label, phase 3 trial. Lancet Oncol 2019; 20: 781-794.

26 - Dimopoulos M, Quach H, Mateos M-V et al. Carfilzomib, dexamethasone, and daratumumab versus carfilzomib and dexamethasone for patients with relapsed or refractory multiple myeloma: results from a randomized, multicenter, open-label, phase 3 study. Lancet. 2020 Jul 18;396(10245):186-197. doi: 10.1016/S01406736(20)30734-0.

27 - Moreau P, Dimopoulos $\mathrm{M}$, Mikhael $\mathrm{J}$ et al. Isatuximab plus carfilzomib and dexamethasone in relapsed/refractory multiple myeloma (IKEMA): interim analysis of a phase 3, randomized, open-label study. EHA Library 2020; LB2603.

28 - Jakubowiak A, Offidani M, Pégourie B, De La Rubia J, Garderet L, Laribi K et al. Randomized phase 2 study: elotuzumab plus bortezomib/dexamethasone vs bortezomib/dexamethasone for relapsed/refractory MM. Blood 2016; 127: 28332840.

29 - Chari A, Suvannasankha A, Fay JW et al. Daratumumab plus pomalidomide and dexamethasone in relapsed and/or refractory multiple myeloma. Blood 2017; 130: 974-981.

30 - Siegel DS, Schiller GJ, Samaras CJ, et al. Pomalidomide, dexamethasone, and daratumumab in relapsed refractory multiple myeloma after lenalidomide treatment.. Leukemia. 2020 May 6. doi: 10.1038/s41375-020-0813-1. Online ahead of print. Leukemia. 2020. PMID: 32376855

31 - Sonneveld P, Zweegman S, Cavo M, Nasserinejad K, Troia R, Pour L et al. Carfilzomib, Pomalidomide and Dexamethasone (KPd) in Patients with Multiple 
Myeloma Refractory to Bortezomib and Lenalidomide. the EMN011 Trial. Blood. 2018, pp 801-801.

32 - Krishnan A, Kapoor P, Palmer JM, Tsai NC, Kumar S, Lonial S, et al. Phase I/II trial of the oral regimen ixazomib, pomalidomide, and dexamethasone in relapsed/refractory multiple myeloma. Leukemia. 2017 Dec 18. doi: 10.1038/leu.2017.352. [Epub ahead of print]

33 - Siegel DS, Dimopoulos MA, Ludwig H, Facon T, Goldschmidt H, Jakubowiak A, et al. Improvement in Overall Survival With Carfilzomib, Lenalidomide, and Dexamethasone in Patients With Relapsed or Refractory Multiple Myeloma. J Clin Oncol. 2018 Mar 10;36(8):728-734. doi: 10.1200/JCO.2017.76.5032. Epub 2018 Jan 17.

34 - Dimopoulos M, Lonial S, White D, et al. Elotuzumab, lenalidomide and dexamethasone in RRMM: final overall survival results from the phase 3 randomized ELOQUENT-2 study. Blood Cancer J 2020, in press

35 - Bahlis NJ, Dimopoulos MA, White DJ, Benboubker L, Cook G, Leiba M, et al. Daratumumab plus lenalidomide and dexamethasone in relapsed/refractory multiple myeloma: extended follow-up of POLLUX, a randomized, open-label, phase 3 study. Leukemia. 2020 Jan 30. doi: 10.1038/s41375-020-0711-6. [Epub ahead of print]

36 - Mateos MV, Nahi H, Legiec W, Grosicki S, Vorobyev V, Spicka I, et al. Subcutaneous versus intravenous daratumumab in patients with relapsed or refractory multiple myeloma (COLUMBA): a multicentre, open-label, non-inferiority, randomised, phase 3 trial. Lancet Haematol. 2020 Mar 23. pii: S23523026(20)30070-3. doi: 10.1016/S2352-3026(20)30070-3. [Epub ahead of print]

37 - Dimopoulos MA, Delimpasi S, Simonova M, et al. Weekly selinexor, bortezomib, and dexamethasone (SVd) versus twice weekly bortezomib and dexamethasone (Vd) in patients with multiple myeloma (MM) after one to three prior therapies: Initial results of the phase III BOSTON study. J Clin Oncol 38, no. 15_suppl (May 20, 2020) 8501-8501. DOI: $10.1200 / J C O .2020 .38 .15 \_s u p p l .8501$

38 - Mateos MV, Dimopoulos MA, Cavo M et al. Daratumumab plus Bortezomib, Melphalan, and Prednisone for Untreated Myeloma. N Engl J Med 2018; 378: 518528.

39 - Mateos MV, Cavo M, Blade $\mathrm{J}$ et al. Overall survival with daratumumab, bortezomib, melphalan, and prednisone in newly diagnosed multiple myeloma (ALCYONE): a randomised, open-label, phase 3 trial. Lancet 2020; 395: 132-141.

40 - Facon T, Kumar S, Plesner $\mathrm{T}$ et al. Daratumumab plus Lenalidomide and Dexamethasone for Untreated Myeloma. N Engl J Med 2019; 380: 2104-2115. 
41 - Attal M, Lauwers-Cances V, Hulin C et al. Lenalidomide, Bortezomib, and Dexamethasone with Transplantation for Myeloma. N Engl J Med 2017; 376: 1311 1320.

42 - Cook G, Williams C, Brown JM, Cairns DA, Cavenagh J, Snowden JA, et al. High-dose chemotherapy plus autologous stem-cell transplantation as consolidation therapy in patients with relapsed multiple myeloma after previous autologous stemcell transplantation (NCRI Myeloma X Relapse [Intensive trial]): a randomised, openlabel, phase 3 trial. Lancet Oncol. 2014 Jul;15(8):874-85. doi: 10.1016/S14702045(14)70245-1. Epub 2014 Jun 16. Erratum in: Lancet Oncol. 2014 Aug;15(9):e365. Dosage error in article text.

43 - Cook G, Ashcroft AJ, Cairns DA, Williams CD, Brown JM, Cavenagh JD, et al. The effect of salvage autologous stem-cell transplantation on overall survival in patients with relapsed multiple myeloma (final results from BSBMT/UKMF Myeloma $X$ Relapse [Intensive]): a randomised, open-label, phase 3 trial. Lancet Haematol. 2016 Jul;3(7):e340-51. doi: 10.1016/S2352-3026(16)30049-7.

44 - Salvage autologous transplant and lenalidomide maintenance vs. lenalidomide/dexamethasone for relapsed multiple myeloma: the randomized GMMG phase III trial ReLApsE. Leukemia.2020 Jul 21.doi:10.1038/s41375-020-09480 . Online ahead of print.

45 - San Miguel J, Weisel K, Moreau P, et al. Pomalidomide plus low-dose dexamethasone versus high-dose dexamethasone alone for patients with relapsed and refractory multiple myeloma (MM-003): a randomised, open-label, phase 3 trial. Lancet Oncol. 2013;14(11):1055-1066.

46 - Attal M, Richardson PG, Rajkumar SV et al. Isatuximab plus pomalidomide and low-dose dexamethasone versus pomalidomide and low-dose dexamethasone in patients with relapsed and refractory multiple myeloma (ICARIA-MM): a randomised, multicentre, open-label, phase 3 study. Lancet 2019; 394: 2096-2107.

47 - Dimopoulos MA, Dytfeld D, Grosicki S et al. Elotuzumab plus Pomalidomide and Dexamethasone for Multiple Myeloma. N Engl J Med 2018; 379: 1811-1822.

48 - Baz RC, Martin TG, Lin HY, Zhao X, Shain KH, Cho HJ, Wolf JL, et al. Randomized multicenter phase 2 study of pomalidomide, cyclophosphamide, and dexamethasone in relapsed refractory myeloma. Blood. 2016 May 26;127(21):25618. doi: 10.1182/blood-2015-11-682518. Epub 2016 Mar 1

49 - Gandhi UH, Cornell RF, Lakshman A et al. Outcomes of patients with multiple myeloma refractory to CD38-targeted monoclonal antibody therapy. Leukemia 2019; 33: 2266-2275. 
50 - Kapoor P, Ramakrishnan V, Rajkumar SV. Bortezomib combination therapy in multiple myeloma. Kapoor P, Ramakrishnan V, Rajkumar SV. Semin Hematol. 2012 Jul;49(3):228-42. doi: 10.1053/j.seminhematol.2012.04.010.

51 - Chari A, Vogl DT, Gavriatopoulou M et al. Oral Selinexor-Dexamethasone for Triple-Class Refractory Multiple Myeloma. N Engl J Med 2019; 381: 727-738.

52 - Richardson PG, Schlossman RL, Alsina M, Weber DM, Coutre SE, Gasparetto $\mathrm{C}$, et al. PANORAMA 2: panobinostat in combination with bortezomib and dexamethasone in patients with relapsed and bortezomib-refractory myeloma. Blood. 2013 Oct 3;122(14):2331-7. doi: 10.1182/blood-2013-01-481325. Epub 2013 Aug 15.

53 - Richardson PG, Bringhen S, Voorhees P, Plesner T, Mellqvist UH, Reeves B, et al. Melflufen plus dexamethasone in relapsed and refractory multiple myeloma (O-12M1): a multicentre, international, open-label, phase 1-2 study. Lancet Haematol. 2020 Mar 23. pii: S2352-3026(20)30044-2. doi: 10.1016/S2352-3026(20)30044-2. [Epub ahead of print]

54 - Tai YT, Anderson KC. B cell maturation antigen (BCMA)-based immunotherapy for multiple myeloma. Expert Opin Biol Ther. 2019 Nov;19(11):1143-1156. doi: 10.1080/14712598.2019.1641196. Epub 2019 Jul 11.

55 - Lonial S, Lee HC, Badros A et al. Belantamab mafodotin for relapsed or refractory multiple myeloma (DREAMM-2): a two-arm, randomised, open-label, phase 2 study. Lancet Oncol 2020; 21: 207-221.

56 - Trudel S, Lendvai N, Popat R, Voorhees PM, Reeves B, Libby EN, et al. Antibody-drug conjugate, GSK2857916, in relapsed/refractory multiple myeloma: an update on safety and efficacy from dose expansion phase I study. Blood Cancer J. 2019 Mar 20;9(4):37. doi: 10.1038/s41408-019-0196-6

57 - Raje N, Berdeja J, Lin Y et al. Anti-BCMA CAR T-Cell Therapy bb2121 in Relapsed or Refractory Multiple Myeloma. N Engl J Med 2019; 380: 1726-1737.

58 - Munshi N, Anderson LD, Shah N, et al. Idecabtagene vicleucel (ide-cel, bb2121), a BCMA-targeted CAR T cell therapy, in patients with relapsed and refractory multiple myeloma (RRMM): initial KarMMa results. J Clin Oncol 2020; 38, no. 15_suppl (May 20, 2020) 8503-8503

59 - Xu J, Chen LJ, Yang SS, Sun Y, Wu W, Liu YF, et al. Exploratory trial of a biepitopic CAR T-targeting $B$ cell maturation antigen in relapsed/refractory multiple myeloma. Proc Natl Acad Sci U S A. 2019 May 7;116(19):9543-9551. doi: 10.1073/pnas.1819745116. Epub 2019 Apr 15.

60 - Berdeja GJ, Madduri D, Usmani SZ, et al. Update of CARTITUDE-1: A phase $1 \mathrm{~b} / 2$ study of JNJ-4528, a B-cell maturation antigen (BCMA)-directed CAR-T cell 
therapy, in relapsed/refractory multiple myeloma. J Clin Oncol 2020; 38, no. 15_suppl (May 20, 2020) 8505-8505.

61 - Sidana S, Shah N. CAR T-cell therapy: is it prime time in myeloma? Hematology Am Soc Hematol Educ Program. 2019 Dec 6;2019(1):260-265. doi: 10.1182/hematology.2019000370.

62 - Moreau P, Harrison S, Cavo M, et al. Updated Analysis of Bellini, a Phase 3 Study of Venetoclax or Placebo in Combination with Bortezomib and Dexamethasone in Patients with Relapsed/Refractory Multiple Myeloma. Blood (2019) 134 (Supplement_1): 1888. 